\title{
Intermittency induced by attractor-merging crisis in the Kuramoto-Sivashinsky equation
}

\author{
Erico L. Rempel and Abraham C.-L. Chian \\ National Institute for Space Research (INPE) and World Institute for Space Environment Research (WISER), P. O. Box 515, São José dos \\ Campos, São Paulo, 12227-010, Brazil
}

(Received 23 April 2004; revised manuscript received 14 October 2004)

\begin{abstract}
We characterize an attractor-merging crisis in a spatially extended system exemplified by the KuramotoSivashinsky equation. The simultaneous collision of two coexisting chaotic attractors with an unstable periodic orbit and its associated stable manifold occurs in the high-dimensional phase space of the system, giving rise to a single merged chaotic attractor. The time series of the post-crisis regime display intermittent behavior. The origin of this crisis-induced intermittency is elucidated in terms of alternate switching between two chaotic saddles embedded in the merged chaotic attractor.
\end{abstract}

DOI: XXXX

Chaotic attractors in nonlinear dissipative systems can undergo sudden changes as a system parameter is varied. These discontinuous changes, called crises, occur when the chaotic attractor collides with an unstable periodic orbit (UPO) and its stable manifold [1-6]. There has been a great interest in the study of crises in partial differential equations (PDE's) in recent years. Chian et al. [3] characterized a highdimensional interior crisis in the Kuramoto-Sivashinsky (KS) equation [7]. Rempel and Chian, and Rempel et al. [5] studied the role of nonattracting chaotic sets, called chaotic saddles, in the onset of the interior crisis reported by Chian et al. [3]. Transition to spatiotemporal chaos via crisis in a drift wave system was studied by He and Chian [8]. In this paper we characterize an attractor-merging crisis in the Kuramoto-Sivashinsky equation.

In an attractor-merging crisis two or more chaotic attractors merge to form one single chaotic attractor [2]. At a critical value of the control parameter, the pre-crisis chaotic attractors simultaneously touch the boundary separating their basins of attraction, and collide with one or more UPO's on the basin boundary. Attractor-merging crises have been observed in several numerical [2,9] and experimental [10] works. Most previous works on merging crisis were restricted to low-dimensional dynamical systems [2,9]. Chian et al. [11] characterized an attractor-merging crisis in a van der Pol model of nonlinear business cycles. Sauer et al. [12] reported the occurrence of an attractor merging in a spatially extended system given by an Ikeda-like model. Münkel and Kaiser [13] discovered an intermittent route to chaos via attractor merging in the Laser-Kuramoto-Sivashinsky equation. In this paper we present a detailed characterization of an attractor-merging crisis in the KS equation, by demonstrating the simultaneous collision of two chaotic attractors with an unstable periodic orbit and the basin boundary. The time series of the post-crisis regime display intermittency, which can be seen as an alternation between two chaotic transients due to coupling between two chaotic saddles [5,6,14-16] embedded in the post-crisis chaotic attractor. Hunt et al. [17], and Sweet and Ott [18] derived a formula relating the exit time $\tau$, the dimension of the stable manifolds and the Lyapunov exponents of a chaotic saddle, and verified the validity of the formula for two- (2D) and fourdimensional (4D) dynamical systems. We apply this formula
PACS number(s): 05.45.Pq, 47.20.Ky

to the KS equation and establish a relation between the characteristic time scale of the intermittent regime and the underlying chaotic saddles.

The Kuramoto-Sivashinsky equation can be written as $[3,5,7,19]$

$$
\partial_{t} u=-\partial_{x}^{2} u-\nu \partial_{x}^{4} u-\partial_{x} u^{2}
$$

where $\nu$ is a "viscosity" damping parameter. We assume that $u(x, t)$ is subject to periodic boundary conditions $u(x, t)$ $=u(x+2 \pi, t)$. To obtain the numerical solution of Eq. (1) we use the spectral Galerkin method, by applying a Fourier decomposition for the function $u(x, t), u(x, t)=\sum_{k=-\infty}^{\infty} b_{k}(t) e^{i k x}$, which yields an infinite set of ordinary differential equations for the complex Fourier coefficients $b_{k}(t)$. To simplify the analysis we restrict our attention to the subspace of odd functions $u(x, t)=-u(-x, t)$, and assume $b_{k}(t)$ purely imaginary by setting $b_{k}(t)=-i a_{k}(t) / 2$, where $a_{k}(t)$ are real. The final set of equations is given by $[3,5,19]$

$$
\dot{a}_{k}(t)=\left(k^{2}-\nu k^{4}\right) a_{k}(t)-\frac{k}{2} \sum_{m=-N}^{N} a_{m}(t) a_{k-m}(t),
$$

where the dot denotes derivative with respect to $t$, and 1 $\leqslant k \leqslant N, N$ is the truncation order. We adopt a Poincaré map defined as the $(N-1)$ dimensional hyperplane given by $a_{1}$ $=0$, with $\dot{a}_{1}>0$, and study the dynamics of the KS equation by varying the control parameter $\nu$. Following Refs. [3,5,19], we choose a range of $\nu$ for which the dynamics of the KS equation can be chaotic in time, but the coherent spatial structures are preserved, with a small number of excited modes. In such regimes, the solutions of the KS equation display many features typical of low-dimensional dynamical systems. We choose $N=16$, since numerical results show that the dynamics is qualitatively the same for larger $N$.

A bifurcation diagram for Eq. (2) can be constructed by plotting the value of any given Fourier mode of a randomly initialized trajectory as a function of the control parameter $\nu$. A supercritical Hopf bifurcation (HB) [20] occurs at $\nu=\nu_{H B}$ $\approx 0.03462$. For $\nu>\nu_{H B}$, random initial conditions are attracted to a steady state. For $\nu<\nu_{H B}$, the steady state becomes unstable, and a period-one (P1) stable limit cycle 

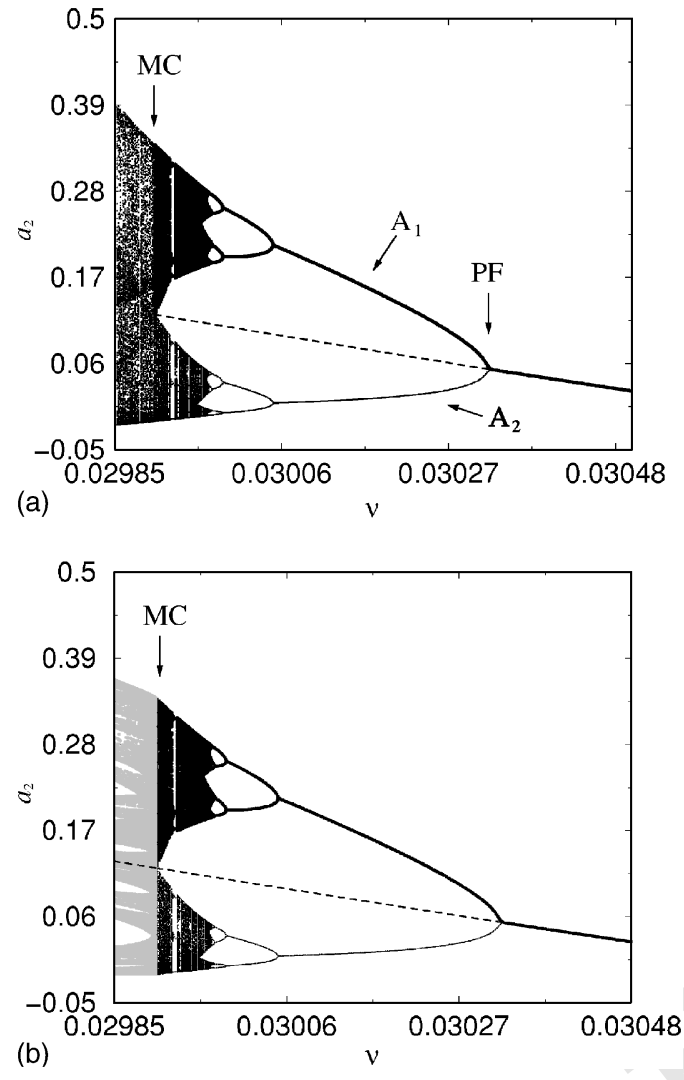

FIG. 1. (a) Superposition of the bifurcation diagrams for attractors $\mathrm{A}_{1}$ (dark lines) and $\mathrm{A}_{2}$ (light lines), showing the merging of these two attractors into a single attractor at MC. PF denotes pitchfork bifurcation, and MC denotes attractor-merging crisis. The dashed line represents the mediating unstable periodic orbit (M) created at PF; (b) conversion of attractors $A_{1}$ and $A_{2}$ into two chaotic saddles (gray) after MC. The two chaotic saddles are separated by the dashed line.

arises. In the Poincaré map this limit cycle appears as a fixed point attractor. At $\nu=\nu_{P F} \approx 0.03031$ the P1 attractor loses its asymptotic stability in a supercritical pitchfork bifurcation (PF) [20], and becomes an unstable periodic orbit (equivalent to a saddle point in the Poincare map) for $\nu<\nu_{P F}$. At the same point, two new $\mathrm{P} 1$ periodic attractors, $\mathrm{A}_{1}$ and $\mathrm{A}_{2}$, are created. The bifurcation diagram of the Fourier mode $a_{2}$ for attractors $\mathrm{A}_{1}$ (dark lines) and $\mathrm{A}_{2}$ (light lines) is shown in Fig. 1(a). The dashed line represents the evolution of the UPO created at PF, and Merging Crisis (MC) denotes attractormerging crisis. Both attractors $\mathrm{A}_{1}$ and $\mathrm{A}_{2}$ undergo a similar sequence of period-doubling bifurcations that leads to two disjoint chaotic attractors. The symmetry between $\mathrm{A}_{1}$ and $\mathrm{A}_{2}$ reflects the invariance under the shift $u(x, t) \rightarrow u(x+\pi, t)$, which is a particular case of the translation invariance property of Eq. (1) under periodic boundary conditions in the subspace of odd functions [19]. At the crisis point $\nu=\nu_{M C}$ $\approx 0.02990058$ the two chaotic attractors simultaneously collide with the $\mathrm{P} 1$ mediating unstable periodic orbit $(\mathrm{M})$ and merge to form a single wide chaotic attractor for $\nu<\nu_{M C}$.

After colliding with $\mathrm{M}$, the two pre-crisis chaotic attractors lose their stability and are converted into two chaotic saddles $\left(\mathrm{CS}_{1}\right.$ and $\left.\mathrm{CS}_{2}\right)$ immersed in the merged chaotic at- tractor, similarly to what happens in the interior crisis $[5,6,16]$. In Fig. 1(b) we plot the bifurcation diagram (black) of Fig. 1(a) up to MC $\left(\nu>\nu_{M C}\right)$, and after MC $\left(\nu<\nu_{M C}\right)$ we plot the variation of the Poincare points of the newly created chaotic saddles (gray). The white space within the gray areas of the chaotic saddles are gaps that reflect the discontinuous and fractal structure of the chaotic saddles along their unstable foliation $[5,6,16]$. $\mathrm{CS}_{1}$ and $\mathrm{CS}_{2}$ are separated by the dashed line in Fig. 1(b).

The characterization of the attractor-merging crisis is done by showing the simultaneous collision of $\mathrm{A}_{1}$ and $\mathrm{A}_{2}$ with the mediating orbit $\mathrm{M}$ and with the boundary separating the basins of attraction of both attractors. The collision of $\mathrm{A}_{1}$ and $\mathrm{A}_{2}$ with $\mathrm{M}$ at $\nu_{M C}$ is displayed in Fig. 2, in which a two-dimensional projection of the Poincaré map $\left(a_{2}, a_{3}\right)$ is adopted. Figure 2(a) shows attractors $\mathrm{A}_{1}$ (dark line) and $\mathrm{A}_{2}$ (light line), and the Poincaré point of the P1 mediating orbit (cross) before the crisis, at $\nu=0.02994$. At $\nu_{M C}$ both attractors collide headon with $\mathrm{M}$, as shown in Fig. 2(b). For $\nu$ $\left\langle\nu_{M C}\right.$ there is only one merged chaotic attractor that contains both pre-crisis attractors.

Next, we show the collision of $\mathrm{A}_{1}$ and $\mathrm{A}_{2}$ with the boundary of their basins of attraction. Since we are working in a 15-dimensional (15D) Poincaré phase space, in order to visualize this collision we choose a suitable $2 \mathrm{D}$ projection of the high-dimensional basins by using a grid of initial conditions generated through a linear interpolation between points on the chaotic attractors [5]. Each initial condition is colored according to the basin to which it belongs. Figure 2(c) is an enlargement of the rectangular region of Fig. 2(b), showing the basins of attraction for attractors $\mathrm{A}_{1}$ and $\mathrm{A}_{2}$ at $\nu_{M C}$. The dark background represents points in the basin of $A_{1}$ (dark lines) and the white background represents the basin of $\mathrm{A}_{2}$ (light line). At crisis both attractors collide with their common basin boundary, as well as with the mediating orbit $\mathrm{M}$ (cross). The boundary is also the stable manifold of $\mathrm{M}$.

After crisis, an orbit in the chaotic attractor can spend some transient time in the region previously occupied by the pre-crisis $A_{1}$ attractor (region 1), before it crosses the stable manifold of $\mathrm{M}$ and moves into the region of the pre-crisis $\mathrm{A}_{2}$ attractor (region 2). It then spends another transient time in region 2 , before moving back to region 1 . This dynamics repeats intermittently, leading to crisis-induced intermittency [2]. Figure 3(a) shows an intermittent time series with the Poincaré points of the Fourier mode $a_{2}$ at $\nu=0.02990$, after crisis. Figure 3(b) shows the space-time contour plot for the intermittent regime at $\nu=0.02990$.

The characteristic intermittency time, defined as the average time between switches among regions 1 and 2, is closely related to the two underlying chaotic saddles present in the post-crisis chaotic attractor CA, shown in Fig. 4(a) for $\nu$ $=0.02987$. After the merging crisis the two pre-crisis chaotic attractors $A_{1}$ and $A_{2}$ lose their asymptotic stability, since typical orbits no longer stay in regions 1 or 2 for all time. However, there are some "atypical" chaotic orbits that stay confined in regions 1 and 2 forever. These orbits belong to the two chaotic saddles created after the merging crisis and are depicted in Fig. 4(b). The two chaotic saddles, $\mathrm{CS}_{1}$ (dark lines) and $\mathrm{CS}_{2}$ (light lines), are found with the pim triple algorithm [15], and are subsets of the post-crisis chaotic at- 



FIG. 2. Two-dimensional projection $\left(a_{2}, a_{3}\right)$ of attractors $\mathrm{A}_{1}$ (dark lines) and $\mathrm{A}_{2}$ (light lines): (a) before the merging crisis; and (b) at the merging crisis point, $\nu_{M C}$. The cross denotes the mediating orbit; (c) is an enlargement of the rectangular region in (b), showing the basins of attraction for $\mathrm{A}_{1}$ (dark lines) and $\mathrm{A}_{2}$ (light line) at $\nu_{M C}$. The dark background represents the basin of $\mathrm{A}_{1}$ and the white region represents the basin of $\mathrm{A}_{2}$.

tractor. The orbits of all initial conditions in the former basin of $A_{1}$ (basin of $A_{2}$ ) eventually leave this region, moving in to region 2 (region 1), except for initial conditions on the stable manifold of $\mathrm{CS}_{1} \mathrm{CS}_{2}$, which is a set of measure zero. All the other trajectories wander in the close vicinity of $\mathrm{CS}_{1} \mathrm{CS}_{2}$ for some transient time, before leaving to region 2 (region 1 ). These transitions between regions 1 and 2 are due to the coupling UPO's that are located within the gaps of $\mathrm{CS}_{1}$ and $\mathrm{CS}_{2}$, and establish the dynamical connection between the two chaotic saddles $[6,16]$.

Let $\tau$ denote the average exit time a random initial condition takes to leave the vicinity of a chaotic saddle. Hunt $e t$ $a l$. [17] and Sweet and Ott [18] derived the following for-
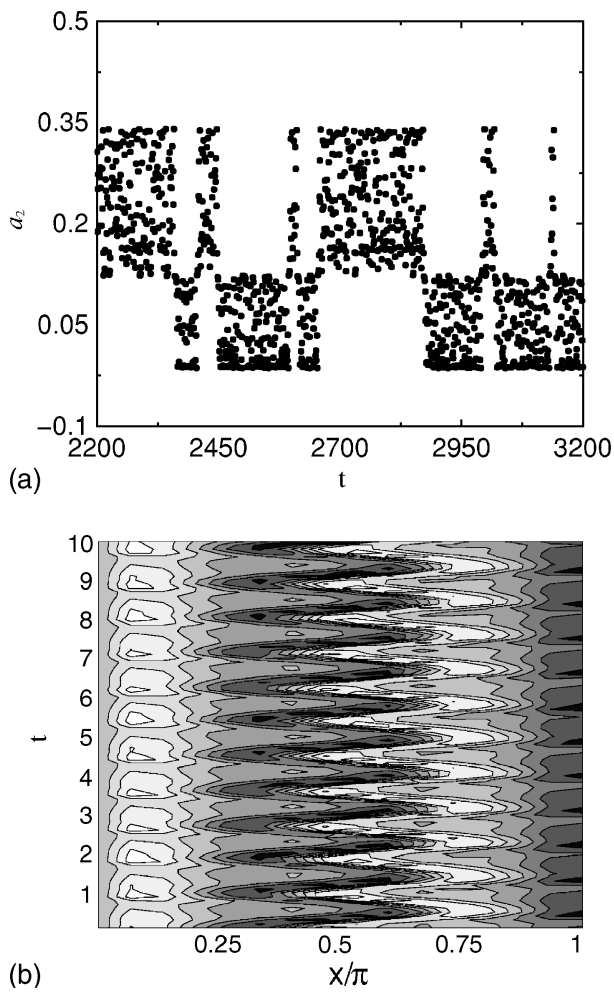

FIG. 3. (a) Intermittent time series of $a_{2}$ at $\nu=0.02990$; (b) contour plot of the space-time evolution of the intermittent regime at $\nu=0.02990$, showing spatial coherence.

mula relating $\tau$, the Lyapunov exponents and the fractal dimension of the stable manifolds of a chaotic saddle. Suppose the system is an $N$-dimensional map, with $U$ positive and $S$ negative Lyapunov exponents, such that $U+S=N$. The exponents can be labeled as $\lambda_{U}^{+} \geqslant \lambda_{U-1}^{+} \geqslant \cdots \geqslant \lambda_{1}^{+}>0>-\lambda_{1}^{-}$ $\geqslant-\lambda_{2}^{-} \geqslant \cdots \geqslant-\lambda_{S}^{-}$. Then, the fractal dimension $\left(D_{s}\right)$ of the stable manifold of a chaotic saddle is given by

$$
\begin{aligned}
D_{s}= & S+J \\
& +\left[\left(\sum_{j=1}^{U} \lambda_{j}^{+}-1 / \tau\right)-\left(\lambda_{1}^{+}+\lambda_{2}^{+}+\cdots+\lambda_{J}^{+}\right)\right] / \lambda_{J+1}^{+},
\end{aligned}
$$

where $J$ is defined by $\lambda_{1}^{+}+\cdots+\lambda_{J}^{+}+\lambda_{J+1}^{+} \geqslant\left(\sum_{j=1}^{U} \lambda_{j}^{+}-1 / \tau\right)$ $\geqslant \lambda_{1}^{+}+\cdots+\lambda_{J}^{+}$.

We use Eq. (3) to compute the average exit time $\tau$. The Lyapunov spectra are computed from pim-triple trajectories. The convergence of the maximum Lyapunov exponent $\lambda_{\max }$ $\approx 0.76$ for $\nu=0.02990$ is shown in Fig. 5(a). There is one null exponent (representing the direction along the flow) and 14 negative exponents, so that $S=14, U=1$, and $J=0$. The value of $D_{s}$ is found with the following version of the uncertainty algorithm for box counting dimension [21,22]: randomly choose an initial condition $x$ on a one-dimensional line inside the former basin of attractor $A_{1}$. If the exit time of $x$ (i.e., the time the orbit of $x$ takes to escape from region 1) is greater than the average exit time $\tau$, then consider $x$ be- 


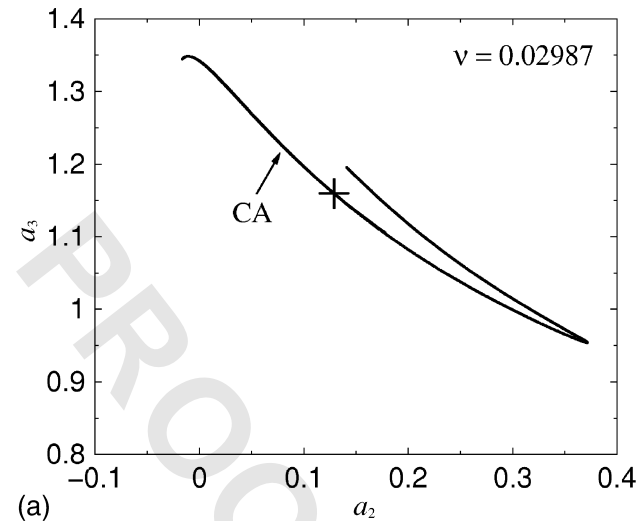

(a)

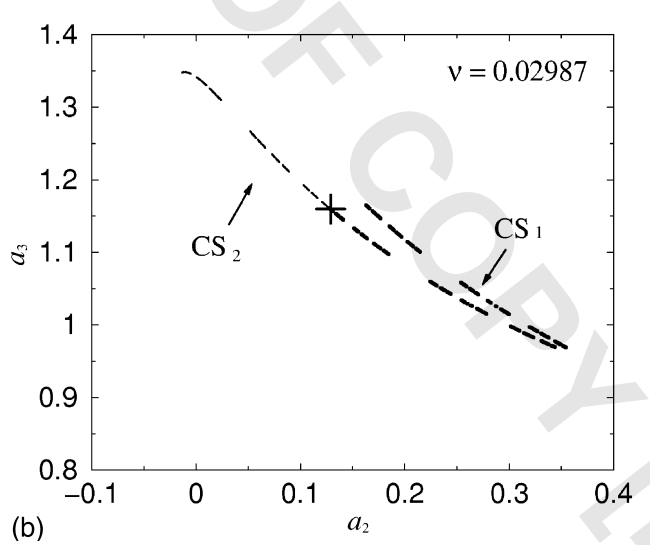

FIG. 4. (a) Post-crisis chaotic attractor (CA); (b) the two chaotic saddles, $\mathrm{CS}_{1}$ and $\mathrm{CS}_{2}$, that compose $\mathrm{CA}$.

longs to the stable manifold (SM) of $\mathrm{CS}_{1}$. Apply a small perturbation $\epsilon$ to $x$. If one of the three points, $x, x+\epsilon$, and $x-\epsilon$, belongs to SM and another does not belong to SM, then $x$ is called uncertain. Compute the fraction of uncertain points $f(\epsilon)$ for a large number of initial conditions $x$. For fractal sets the value of $f(\epsilon)$ scales with $\epsilon$ as $f(\epsilon) \sim \epsilon^{\alpha}$, where $\alpha$ is the uncertainty exponent. Figure 5(b) illustrates the computation of $\alpha$ from the graph of $f(\epsilon)$ versus $\epsilon$ on a base-10 logarithmic scale for $\nu=0.02990$. The slope of the fitted line gives the uncertainty exponent $\alpha \approx 0.018$. The boxcounting dimension of the intersection of the stable manifold with the 1D line is $d_{s}=1-\alpha$. In the full 15D phase space of the Poincaré map, the box-counting dimension of the stable manifold of the chaotic saddle is $D_{s}=15-\alpha=14+d_{s}$ [18].

We compare the values of $\tau$ obtained from Eq. (3) with the characteristic intermittency time (also denoted by $\tau$ ), obtained as the average over a long time series of the switching time between regions 1 and 2. Figure 6 is a plot of $\ln _{10} \tau$ vs $\ln _{10}\left(\nu_{M C}-\nu\right)$, where the solid line with slope $\gamma \approx-0.52$ is a linear fit of the values of the characteristic intermittency time computed from time series (circles). Figure 6 reveals that the characteristic time $\tau$ decreases with the distance from the critical parameter value $\nu_{M C}$ following a power-law decay, $\tau \sim\left(\nu_{M C}-\nu\right)^{\gamma}$, as expected [2]. The crosses denote the exit time from $\mathrm{CS}_{1}$ computed from Eq. (3) (the value of $\tau$ is the same for $\mathrm{CS}_{1}$ and $\mathrm{CS}_{2}$, due to the symmetry of the problem). The agreement between the values of $\tau$ obtained from time series and from Eq. (3) demonstrates that the characteristic
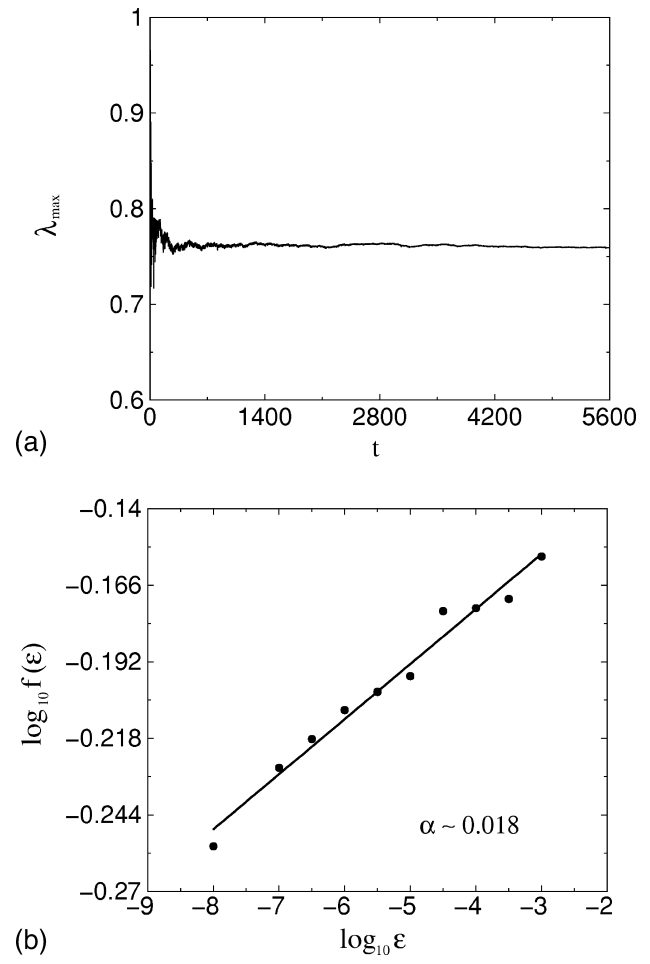

FIG. 5. (a) Convergence of the maximum Lyapunov exponent of the chaotic saddle at $\nu=0.02990$; (b) Plot of the fraction of uncertain initial conditions $f(\epsilon)$ vs the uncertainty $\epsilon$ on a base-10 logarithmic scale. The slope of the fitted line gives the uncertainty exponent $\alpha \approx 0.018$. In the 15 -dimensional phase space of the Poincare map, the fractal dimension of the stable manifold of the chaotic saddle is $D_{s}=15-\alpha \approx 14.968$.

time scale of this crisis-induced intermittency can be predicted from the intrinsic properties of the chaotic saddles. Alternatively, the value of $\gamma$ can be found by using the value of $\tau$ obtained from Eq. (3) and the relation $\tau \sim\left(\nu_{M C}-\nu\right)^{\gamma}$. For example, for $\nu=0.02990$ we find $\gamma \approx-0.51$, which is close to the value obtained from time series $(\gamma \approx-0.52)$.



FIG. 6. $\ln _{10} \tau$ vs $\ln _{10}\left(\nu_{M C}-\nu\right)$. The solid line with slope $\gamma$ $\approx-0.52$ is a linear fit of the values of the characteristic intermittency time $\tau$ computed from time series (circles); the crosses denote the exit time from $\mathrm{CS}_{1}$ computed with Eq. (3). 
In summary, we have characterized an attractor-merging crisis in a partial differential equation exemplified by the Kuramoto-Sivashinsky equation. In particular, the role of chaotic saddles in the crisis-induced intermittency has been elucidated in this high-dimensional dynamical system. We make use of a relation between the exit time, Lyapunov exponents and stable manifolds of a chaotic saddle to predict the characteristic time scale of the intermittency.

This work is supported by CNPq and FAPESP.
[1] C. Grebogi, E. Ott, and J. A. Yorke, Physica D 7, 181 (1983).

[2] C. Grebogi, E. Ott, F. Romeiras, and J. A. Yorke, Phys. Rev. A 36, 5365 (1987).

[3] A. C.-L. Chian, E. L. Rempel, E. E. Macau, R. R. Rosa, and F. Christiansen, Phys. Rev. E 65, 035203(R) (2002).

[4] A. C.-L. Chian, F. A. Borotto, and E. L. Rempel, Int. J. Bifurcation Chaos Appl. Sci. Eng. 12, 1653 (2002); F. A. Borotto, A. C.-L. Chian, and E. L. Rempel, ibid. 14, 2375 (2004); F. A. Borotto, A. C.-L. Chian, T. Hada, and E. L. Rempel, Physica D 194, 275 (2004).

[5] E. L. Rempel and A. C.-L. Chian, Phys. Lett. A 319, 104 (2003); E. L. Rempel, A. C.-L. Chian, E. E. Macau, and R. R. Rosa, CHAOS (to be published).

[6] E. L. Rempel and A. C.-L. Chian, Int. J. Bifurcation Chaos (to be published); E. L. Rempel, A. C.-L. Chian, E. E. Macau, and R. R. Rosa, Physica D (to be published).

[7] R. E. LaQuey, S. M. Mahajan, P. H. Rutherford, and W. M. Tang, Phys. Rev. Lett. 34, 391 (1975); Y. Kuramoto and T. Tsuzuki, Prog. Theor. Phys. 55, 356 (1976); G. I. Sivashinsky, Acta Astron. 4, 1177 (1977).

[8] Kaifen He and A. C.-L. Chian, Phys. Rev. Lett. 91, 034102 (2003); Phys. Rev. E 69, 026207 (2004).

[9] C. L. Pando, G. Perez, and H. A. Cerdeira, Phys. Rev. E 48, 196 (1993); S. Matyjaśkiewicz, A. Krawiecki, J. A. Holyst, K. Kacperski, and W. Ebeling, ibid. 63026215 (2001); S. K. Dwivedy and R. C. Kar, Int. J. Non-Linear Mech. 38, 585 (2003).
[10] A. Magauer and S. Banerjee, IEEE Trans. Circuits Syst., I: Fundam. Theory Appl. 47, 254 (2000); V. N. Chizhevsky, R. Vilaseca, and R. Corbalán, Phys. Rev. E 61, 6500 (2000).

[11] A. C.-L. Chian, F. A. Borotto, E. L. Rempel, and C. Rogers, Chaos, Solitons Fractals (to be published).

[12] M. Sauer, J. Leonardy, and F. Kaiser, Chaos, Solitons Fractals 4, 1461 (1994).

[13] M. Münkel and F. Kaiser, Physica D 98, 156 (1996).

[14] G.-H. Hsu, E. Ott, and C. Grebogi, Phys. Lett. A 127, 199 (1988).

[15] H. E. Nusse and J. A. Yorke, Physica D 36, 137 (1989).

[16] K. G. Szabó, Y.-C. Lai, T. Tél, and C. Grebogi, Phys. Rev. Lett. 77, 3102 (1996); K. G. Szabó, Y.-C. Lai, T. Tél, and C. Grebogi, Phys. Rev. E 61, 5019 (2000).

[17] B. R. Hunt, E. Ott, and J. A. Yorke, Phys. Rev. E 54, 4819 (1996).

[18] D. Sweet and E. Ott, Physica D 139, 1 (2000).

[19] F. Christiansen, P. Ćvitanovic, and V. Putkaradze, Nonlinearity 10, 55 (1997).

[20] J. Guckenheimer and P. Holmes, Nonlinear Oscillations, Dynamical Systems, and Bifurcations of Vector Fields (Springer, New York, 1983).

[21] C. Grebogi, S. W. McDonald, E. Ott, and J. A. Yorke, Phys. Lett. 99A, 415 (1983).

[22] Y.-C. Lai and R. L. Winslow, Phys. Rev. Lett. 74, 5208 (1995). 